\title{
Evidence for interspecific interactions in the ectoparasite infracommunity of a wild mammal
}

\author{
Sasha Hoffmann ${ }^{1}$, Ivan G. Horak², Nigel C. Bennett ${ }^{1}$ and Heike Lutermann ${ }^{1 *}$ (D)
}

\begin{abstract}
Background: Co-infection with multiple parasite species is commonly observed in nature and interspecific interactions are likely to occur in parasite infracommunities. Such interactions may affect the distribution of parasites among hosts but also the response of infracommunities to perturbations. However, the response of infracommunities to perturbations has not been well studied experimentally for ectoparasite communities of small mammal hosts.

Methods: In the current study we used experimental perturbations of the ectoparasite infracommunity of sengis from Africa. We suppressed tick recruitment by applying an acaride and monitored the effects on the ectoparasite community.

Results: Our treatment affected the target as well as two non-target species directly. The experimental removal of the dominant tick (Rhipicephalus spp.) resulted in increases in the abundance of chiggers and lice. However, while these effects were short-lived in chiggers, which are questing from the environment, they were long-lasting for lice which spend their entire life-cycle on the host. In addition, the recruitment rates of some ectoparasite species were high and did not always correspond to total burdens observed.

Conclusion: These findings indicate that infracommunity interactions may contribute to patterns of parasite burdens. The divergent responses of species with differing life-history traits suggest that perturbation responses may be affected by parasite life-history and that the ectoparasite infracommunity of sengis may lack resilience to perturbations. The latter observation contrasts with the high resilience reported previously for endoparasite communities and also suggests that anti-parasite treatments can affect the distribution of non-target species.
\end{abstract}

Keywords: Community ecology, Co-infection, Ectoparasite, Interaction, Rhipicephalus

\section{Background}

The distribution of parasites depends on the exposure and susceptibility of hosts in a population $[1,2]$. However, in nature it is rarely a single parasite species that infests an individual host and parasites co-infecting a host can be expected to interact with each other $[3,4]$. The nature of such interactions can range from antagonistic to facilitating, depending on whether two parasites interact directly (physically or chemically) or indirectly via shared resources (bottom-up regulation) or the host's immune system (top-down regulation) [5, 6]. In turn, such interactions may be expected to affect

\footnotetext{
* Correspondence: hlutermann@zoology.up.ac.za

${ }^{1}$ Department of Zoology and Entomology, University of Pretoria, Private Bag X20, Hatfield 0028, South Africa

Full list of author information is available at the end of the article
}

the susceptibility to other parasites and this has implications for disease ecology and epidemiology [7, 8]. Consequently, the infection with one parasite species may reduce or increase the probability of invasion by another parasite species, affect its subsequent establishment and clearance rate and modulate the morbidity and/or mortality and ultimately transmission rates $[9,10]$.

While interspecific interactions within parasite infracommunities (parasite species assemblage parasitizing a single host) are well documented from laboratory studies $[5,11,12]$, similar studies in wild hosts are often correlational and based on cross-sectional data [7, 13-15]. However, recent theoretical and experimental studies have shown that observational approaches may fail to identify interactions between parasites concomitantly 
infesting a host or incorrectly characterize the type of interaction [16-19]. Consequently, it has been suggested that studies employing experimental manipulations are better suited to identify interspecific relationships between parasite species infesting the same host [18-20].

The resilience (time of recovery from a perturbation) of a community is strongly dependent on the nature and strength of interspecific interactions within a community [21]. These parameters, as well as the complexity of interactions, can in turn determine the effect of perturbations (e.g. removal of a particular species) on the community [21-23]. It has been suggested that these perturbation effects will increase with an increasing number of interactions [21]. In addition, perturbations will result in more long-term effects if they affect keystone species that maintain interactions with many members of the community [24]. However, interspecific interactions and community resilience remain poorly studied in parasite communities and the focus of such studies is biased towards endoparasite communities [7, 13, 14, 17, 19]. Compared to endoparasites our knowledge of the relationships within ectoparasite communities remains limited and largely restricted to within-taxon studies [25-27]. This is despite the complex immunological cascades that some of these ectoparasite species trigger in their hosts $[28,29]$. The limited period that the majority of ectoparasitic arthropods spend on a host [30] compared to many endoparasites species may partially account for this bias in the literature. However, ectoparasitic arthropods such as ticks are important vectors for a variety of pathogens of medical and veterinary significance and acaricides are widely used in the livestock and pet industry to reduce tick infestation [28]. Through interspecific interactions the drug-related reduction of tick prevalence and/or abundance could also affect other (non-target) parasite species with potentially important implications for disease ecology.

In the current study we combine observational data and experimental perturbations to study interspecific relationships in the ectoparasite infracommunity of eastern rock sengis (Elephantulus myurus) in South Africa. They are small (45-80 g), insectivorous mammals that are widely distributed throughout sub-Saharan Africa [31]. Sengis are one of the dominant species in endemic small mammal communities and sustain a diverse ectoparasite community [32, 33]. The aim of the current study was (i) to assess the prevalence and nature of interactions within their ectoparasite infracommunities, (ii) to determine the exposure of hosts to the various ectoparasite taxa in the environment and (iii) to evaluate the response of the infracommunity to experimental perturbation. We used the acaricide Frontline ${ }^{\oplus}$ (fipronil $10 \% \mathrm{w} /$ v/ (s)-methoprene; Merial Pty, Ltd, South Africa) to reduce tick (target parasites) burdens and recruitment.
Ticks were chosen as target taxon since they are the most prevalent and abundant ectoparasite taxon sustained by sengis [33]. As haematophagous parasites they not only deplete host resources directly but also trigger well-known immune responses [28]. All of these characteristics make them a likely taxon to interact with several other members of the sengi ectoparasite community either directly or indirectly via resource depletion (i.e. blood) or the immune responses of the host. Consequently, we (i) expected to find evidence for competitive interactions between ticks and other ectoparasite taxa they might physically interact with such as when competing for attachment sites. In addition, we hypothesized that (ii) competitive interactions between ticks and other haematophagous ectoparasites such as lice would be apparent due to competition for resources such as blood.

\section{Methods}

\section{Collection of animals}

Sengis were captured in eight plots at Goro Game Reserve $\left(22^{\circ} 58^{\prime} \mathrm{S} ; 2^{\circ} 25^{\prime} \mathrm{E}\right)$ in the Limpopo Province, South Africa. Between March 2012 and April 2013 sengis were sampled during six trips (March/April 2012: autumn 12, June 2012: early winter, August 2012: late winter, October 2012: spring, January/February 2013: summer and March/April 2013: autumn 13). Each site was sampled for three nights every second night (Additional file 1: Figure S1) using 150 Sherman traps (H. B. Sherman Traps, Inc. Tallahassee, Florida, U.S.A.) baited with a mixture of sardines, oats and peanut butter. Traps were arranged in $3 \times 50$ grids with approximately 10 paces between neighbouring traps. Due to the uneven terrain of this grid, this layout had to be adjusted for two of our sites (Additional file 1: Figure S2). Captured individuals were sexed, hand-restrained and all ectoparasites encountered during thorough searches of the entire body were removed with fine-tipped forceps. No ectoparasite eggs were removed during this procedure. The parasites collected were stored in $70 \%$ ethanol for later preparation and identification. Sengis were then given unique ear clips for long-term identification. All animals were released at the point of capture in the afternoon.

\section{Experimental manipulation}

Half of the animals caught were treated against ectoparasites by applying Frontline ${ }^{\bullet}$ with the active component fipronil which kills fleas and ticks. Frontline ${ }^{\bullet}$ was sprayed on the handler's gloves and then rubbed over the animal's body as indicated by the supplier. Externally applied it attaches to hair follicles in the dermal skin layer within $24 \mathrm{~h}$ but does not reach past the dermis [34]. Topical application of fipronil is effective for over 30 days [35]. The main component of Frontline spray is isopropyl alcohol which is highly volatile and evaporates within less than $30 \mathrm{~min}$. 
Fipronil has been successfully applied topically to combat ectoparasite infestations in a number of small mammal species in the wild [36-39]. However, the skin absorption, efficacy and permanency of Fipronil may vary between different species and since such measures were not available for the study species we cannot entirely exclude the possibility that these properties may have slightly deviated for sengis from those reported for other small mammals. Our results (see relevant section) do however, suggest that the treatment was effective. Sengis were randomly assigned to the Frontline ${ }^{\circ}$ treatment group to achieve an even distribution between the sexes and sites. Once assigned to a treatment group, individuals remained in this treatment group throughout the entire study. The treatment was applied once per trip for each individual irrespective of whether a particular individual was captured for the first time during the first, second or third night on a particular plot during a trip. In addition, an ectoparasite assessment was conducted for each individual during each recapture within the same trip. Effectively, this approach resulted in two treatments: firstly total ectoparasite removal during each capture and secondly manipulation of ectoparasite recruitment rates as a result of the application of Frontline $e^{\circ}$ to half of the individuals captured. However, due to the transient nature of infestation by ectoparasites other than lice [30], the former procedure still allowed an assessment of competitive interactions in the parasite community. At the same time, ectoparasite recruitment rates can serve as a proxy for parasite exposure, a measure notoriously difficult to assess. Mites and lice were cleared and mounted following standard protocols while ticks were identified directly to genus or species-level when possible (for details on identification procedure see [33]). We noted the presence or absence and counted the number of specimens for each parasite taxon using a dissecting microscope.

\section{Statistical analysis}

We calculated the prevalence (proportion of individuals infested) and mean abundance (total number of ectoparasites divided by the number of hosts sampled, Bush et al. [12]) for each parasite taxon. For the analyses we pooled the counts for ticks across stages since the abundance of nymphs was generally too low to permit a meaningful analysis according to life-history stage. Although this was not the case for Rhipicephalus warburtoni the abundance of larvae and nymphs was highly correlated $\left(R_{S}=0.737, p<0.0001\right)$ and both stages are present throughout the year [40]. In addition, the qualitative results for the separate stages corresponded to those for the pooled data. Hence only the latter are reported here. Since ticks of the genus Rhipicephalus other than $R$. warburtoni had an extremely low prevalence and abundance (see result section) we pooled the data for all specimens from this genus. None of the ectoparasite data collected satisfied the criteria for a normal distribution (Shapiro-Wilk Test: $p<0.001$ ) and transformations were unsuccessful. Therefore, we analysed the effect of season, sex, treatment (untreated vs. treated) and capture (see below) on ectoparasite prevalence employing generalised linear mixed models (GLMMs) using a binomial structure with a logit-link function. All two-way interactions were included in the model. To account for repeated sampling of a study plot and individual, the sengi ID nested within site was added as the random effect for all models. GLMMs were run for all ectoparasite taxa exceeding a prevalence of $10 \%$. Posthoc comparisons were carried out using the least significant difference (LSD). However, few significant effects were found for prevalence and those variables that were significant corresponded to those found for parasite abundance (Additional file 1: Table S1). As a consequence these results are not further discussed. The same variables were included in the full GLMMs for an individual's ectoparasite abundance but we used a negative binomial data distribution with a log-link function. In addition, we examined the effects of season, treatment, sex and capture on the species richness (number of ectoparasite taxa) of a sengi individual employing GLMMs with a Poisson distribution and a log-link function. The variable sex (either as main effect or interaction term) was not significant for any of the measures examined $(\mathrm{p} \leq 0.065)$ while results changed when it was not included in the models. Hence model results are reported without the terms including sex as a factor.

The only animals captured during October 2012 were five pregnant females. Since pregnancies markedly affect tick burden [41], data for this season were excluded from the analyses. In order to evaluate the overall patterns of parasite distribution as well as the effects of our perturbation experiment we conducted two separate analyses. Firstly, we analysed the data including only the first capture of each individual during each trip (long-term data). We repeated these analyses including recaptures within the same trip to assess short-term patterns (Additional file 1: Figure S1). This allowed us to evaluate the overall evidence for interspecific interactions as well as its effect on recruitment rates. In addition, the time elapsed between consecutive trips (8-12 weeks) exceeded the period indicated by the manufacturer for the effectiveness of Frontline ${ }^{\circ}$. Thus analysing data for short-time intervals were conducted to evaluate the effectiveness of our treatment. For these short-term analyses, capture indicated that animals were captured for the first, second or third time within a trip. When analysing long-term effects individuals were classified as either new animals when first captured during the course of this study or as recaptures in a subsequent trip (i.e. capture for analyses 
of long-term data). For the short-term data analyses that included recaptures within the same trip the variable 'capture' had three levels for the first, second or third time that an individual was caught within a particular trip. Models were simplified using backward stepwise elimination of non-significant terms beginning with interaction terms to obtain the minimal model. We evaluated the validity of the final model by comparing the results from this approach with those based on model selection based on the Akaike information criterion [42]. To evaluate the contribution of our random factors we used general linear models using the same data distributions and variables as indicated above. However, since with few exceptions (e.g. short-term abundance of Rhipicephalus spp.) omitting the random effects changed the qualitative results of the models. Consequently, we are only reporting the results for GLMMs. All analyses were carried out in SPSS v.22 and results are reported as means \pm standard error (SE).

\section{Results}

\section{Ectoparasite burdens}

A total of 125 animals were caught $(68 \AA, 57$ क ) between one and ten times during the study period (333 captures in total). Nine tick species, belonging to six genera, one mite and one louse species were collected from these animals (Table 1) with individuals harbouring between 0 and 5 parasite species at a time (Additional file 1: Figure S3). Only immature ticks (i.e. larvae and nymphs) were recovered from the animals. Unlike reported for other sengi populations [33, 43], no fleas were found in the study population during the course of the study. Only two of the ticks (Rhipicephalus (R.) warburtoni and Rhipicentor (Rc.) nuttalli), chigger mites and the louse Neolinognathus elephantuli occurred at substantial prevalence or abundance with $R$. warburtoni being the most prevalent (100\% of first captures) and abundant (mean abundance per individual: 259.02 \pm
129.03 for first captures only). With the exception of the mite all of these species prefer sengis as hosts [33]. In contrast, chiggers are host generalists and exploit a wide range of hosts [44].

\section{Treatment effects on parasite distribution}

Lice were the only ectoparasite taxon affected by our treatment when only long-term data were considered and treated animals $(14.8 \pm 7.9)$ sustained a significantly greater abundance than untreated individuals $(2.3 \pm 1.2$, Table 2). In contrast, all ectoparasite taxa except lice showed a significant effect of treatment on their abundance when short-term data were considered (Table 2). While the abundance of the two tick taxa was significantly reduced in treated compared to untreated individuals, the opposite was true for chiggers (Fig. 1). When considering short-term but not long-term data the treatment resulted in a significantly reduced parasite species richness in treated $(1.57 \pm 0.15)$ compared to untreated individuals $(1.34 \pm 0.14$, Table 3$)$.

\section{Treatment effects on ectoparasite recruitment rates}

Although capture had no significant effect on long-term ectoparasite abundance, it significantly affected all four ectoparasite taxa as well as species richness when shortterm data were considered (Tables 2 and 3). For all taxa abundance was significantly higher during the first compared to the second and third capture (LSD: $\mathrm{p} \leq 0.050$, Additional file 1: Figure S4). In addition, it decreased significantly from second to third capture for both ticks (LSD: $\mathrm{p} \leq 0.018$, Additional file 1: Figure S4) but none of the other taxa or species richness (LSD: $p \geq 0.471$ ). Similarly, species richness was significantly lower during the second $(1.2 \pm 0.1$, LSD: $p<0.0001)$ and third $(1.2 \pm 0.3$, LSD: $p=0.006)$ compared to the first capture $(2.1 \pm 0.1$, Table 3 ) while it did not differ significantly between second and third capture (LSD: $p=0.891$ ).

Table 1 Ectoparasite species collected and their infestation parameters on Elephantulus myurus in the Goro Game Reserve

\begin{tabular}{lllll}
\hline Taxon & Species & Total & Prevalence [\%] $(95 \% \mathrm{Cl})$ & Abundance $(95 \% \mathrm{Cl})$ \\
\hline Ticks & Argas brumpti & 3 & $0.6(0.001-0.023)$ & $0.01(0.00-0.02)$ \\
& Amblyoma hebraeum & 8 & $0.5(0.00-0.03)$ & $0.04(0.00-0.11)$ \\
& Amblyoma marmoreum & 5 & $2.3(0.01-0.05)$ & $0.02(0.00-0.05)$ \\
& Haemaphysalis elliptica & 5 & $1.2(0.00-0.04)$ & $0.01(0.00-0.02)$ \\
& Nuttalliella namaqua & 32 & $0.3(0.00-0.02)$ & $0.10(0.00-0.29)$ \\
& Rhipicentor nuttalli & 987 & $48.3(0.43-0.54)$ & $3.11(2.49-3.75)$ \\
& Rhipicephalus arnoldi & 2 & $0.9(0.00-0.03)$ & $0.01(0.00-0.02)$ \\
& Rhipicephalus simus & 11 & $3.3(0.01-0.07)$ & $0.05(0.02-0.09)$ \\
Mites & Rhipicephalus warburtoni & 54187 & $94.9(0.92-0.97)$ & $169.80(152.44-188.52)$ \\
Lice & Trombiculidae (chiggers) & 2713 & $66.2(0.60-0.73)$ & $15.30(11.97-19.74)$ \\
\hline
\end{tabular}


Table 2 Results of the final GLMMs for long- and short-term effects of season, treatment and capture on the ectoparasite abundance of E. myurus

\begin{tabular}{|c|c|c|c|c|c|c|c|c|c|}
\hline & \multirow[t]{2}{*}{ Variable } & \multicolumn{2}{|c|}{ Rhipicephalus spp. } & \multicolumn{2}{|c|}{ Rc. nuttalli } & \multicolumn{2}{|l|}{ chigger } & \multicolumn{2}{|c|}{ N. elephantuli } \\
\hline & & F-value & $p$-value & $F$-value & $p$-value & $F$-value & $p$-value & $F$-value & $p$-value \\
\hline \multirow[t]{6}{*}{ Long-term data } & Season & 5.813 & $<0.0001$ & 2.367 & 0.054 & 5.690 & $<0.0001$ & 0.223 & 0.925 \\
\hline & Treatment & - & - & 0.000 & 0.998 & 0.581 & 0.447 & 9.057 & 0.003 \\
\hline & Capture & - & - & 0.000 & 0.999 & 0.077 & 0.781 & 0.793 & 0.374 \\
\hline & Season*treatment & - & - & 0.720 & 0.579 & 1.371 & 0.246 & 0.562 & 0.690 \\
\hline & Season*capture & - & - & 0.156 & 0.926 & 2.520 & 0.059 & 0.297 & 0.827 \\
\hline & Treatment* capture & - & - & 0.744 & 0.390 & 0.344 & 0.558 & 2.081 & 0.151 \\
\hline \multirow[t]{6}{*}{ Short-term data } & Season & 7.213 & $<0.0001$ & 4.104 & 0.003 & 0.781 & 0.538 & 0.555 & 0.696 \\
\hline & Treatment & 30.678 & $<0.0001$ & 6.911 & 0.009 & 13.711 & $<0.0001$ & 1.261 & 0.262 \\
\hline & Capture & 447.649 & $<0.0001$ & 9.562 & $<0.0001$ & 9.065 & $<0.0001$ & 4.433 & 0.013 \\
\hline & Season*treatment & 1.654 & 0.161 & - & - & 0.547 & 0.702 & 0.277 & 0.893 \\
\hline & Season*capture & 5.524 & $<0.0001$ & 0.560 & 0.810 & 0.348 & 0.946 & 0.206 & 0.990 \\
\hline & Treatment* capture & 30.089 & $<0.0001$ & 3.731 & 0.025 & 9.305 & $<0.0001$ & 3.488 & 0.032 \\
\hline
\end{tabular}

-: factor dropped from the final model

Significant effects are highlighted in bold

For short-term data the interaction between treatment and capture was significant for all four ectoparasite taxa (Fig. 2, Table 1). The abundance during the first capture did not differ significantly between treatments for any of the ectoparasite taxa (LSD: $\mathrm{p} \geq 0.255$ ). In contrast, the mean abundance of Rhipicephalus spp. was significantly lower for treated than untreated sengis for both second and third captures (LSD: $\mathrm{p} \leq 0.0002$, Fig. 2a). However, mean abundance did not differ significantly between treatments for the second and third capture for Rc. nuttalli and lice ( $\mathrm{p} \leq 0.148$, Fig. $2 \mathrm{~b}$ and $\mathrm{d})$. Conversely, the mean chigger abundance was significantly greater for treated compared to untreated animals for second (LSD: $p=0.046$ ) but not third captures (LSD: $p=0.340$, Fig. 2c).
At the same time, among treated individuals abundance differed significantly between all captures for both tick species (LSD: $\mathrm{p} \leq 0.021$, Fig. $2 \mathrm{a}$ and $\mathrm{b}$ ). Similarly, the mean abundance of $N$. elephantuli was significantly greater during the first capture compared to both second and third capture (LSD: $\mathrm{p} \leq 0.050$, Fig. $2 \mathrm{~d}$ ) but not between second and third capture (LSD: $p=0.706$ ) for treated sengis. In contrast, the mean abundance of chiggers did not differ significantly between captures (LSD: $\mathrm{p} \geq 0.554$ ) of treated individuals. Among untreated sengis, the mean abundance of Rhipicephalus spp. decreased significantly between successive captures (LSD: $\mathrm{p} \leq 0.006$, Fig. 2a) while it was significantly lower during second and third capture compared to the first capture

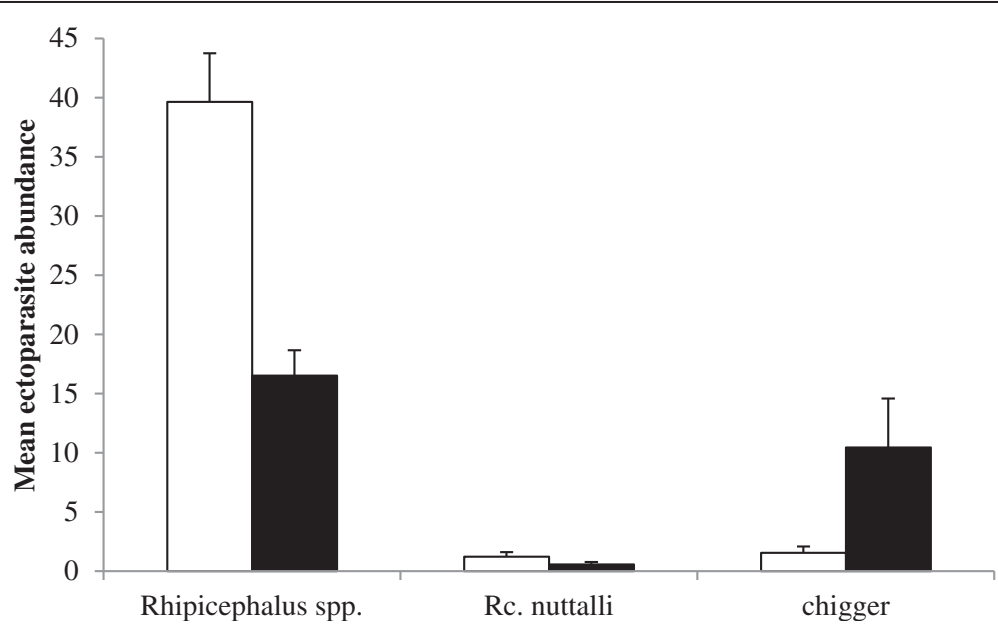

Fig. 1 Effects of treatment on the abundance of Rhipicephalus spp., Rc. nuttalli and chiggers when all capture data are considered. Untreated animals are indicated with open bars while solid bars indicate treated animals. Displayed are means \pm SE 
Table 3 Results of the final GLMMs for long- and short-term effects of season, treatment, capture status, sex on the ectoparasite species richness of $E$. myurus

\begin{tabular}{llllll}
\hline Variable & \multicolumn{2}{l}{ Long-term } & & \multicolumn{2}{l}{ Short-term } \\
\cline { 2 - 3 } & F-value & $p$-value & & F-value & $p$-value \\
\hline Season & 4.216 & $\mathbf{0 . 0 0 3}$ & & 2.115 & 0.079 \\
Treatment & - & - & & 4.013 & $\mathbf{0 . 0 4 6}$ \\
Capture & - & - & 11.450 & $<\mathbf{0 . 0 0 0 1}$ \\
Season*treatment & - & - & 0.284 & 0.971 \\
Season*capture & - & - & - & - \\
Treatment*apture & - & - & - & - \\
\hline
\end{tabular}

-: factor dropped from the final model

Significant effects are highlighted in bold

for Rc. nuttalli and chiggers (LSD: $\mathrm{p} \leq 0.021$; Fig. $2 \mathrm{~b}$ and c) but did not differ significantly between the last two captures (LSD: $\mathrm{p} \geq 0.306$ ). In contrast, mean $N$. elephantuli abundance did not vary significantly between captures for untreated individuals $(p \geq 0.470)$.

\section{Other factors affecting ectoparasite abundance}

The mean long-term abundance of Rhipicephalus spp. and chiggers but not of the other two species differed significantly between seasons (Table 2). For Rhipicephalus spp. it was significantly lower in early winter compared to all other seasons (LSD: $\mathrm{p} \leq 0.025$, Fig. 3a) except late winter (LSD: $p=0.453$ ). Similarly, the mean abundance of Rhipicephalus spp. in late winter was significantly lower than during autumn 12 and autumn 13 (LSD: $\mathrm{p} \leq 0.024$, Fig. 3a) but not summer (LSD: $p=$ 0.089). No other pairwise comparisons were significant. The seasonal variation in chigger abundance differed from that of Rhipicephalus spp. and was significantly lower in late winter compared to all other seasons (LSD: $\mathrm{p} \leq 0.042$ ) except summer (LSD: $p=0.089$, Fig. 3a). In addition, it was significantly greater in autumn 13 compared to autumn 12 (LSD: $p=0.027$, Fig. 3a) while no other pairwise comparisons were significant (LSD: $p \geq$ 0.089). However, the long-term species richness differed significantly between seasons (Table 3 ). It was significantly lower in late winter and spring compared to the other seasons (LSD: $p \leq 0.042$, Additional file 1: Figure S2) while none of the remaining pairwise comparisons were significant (LSD: $\mathrm{p} \geq 0.388$ ).

Season significantly affected the short-term abundance of both ticks but not chiggers and lice (Fig. 3b, Table 2). For both ticks mean abundance was significantly greater

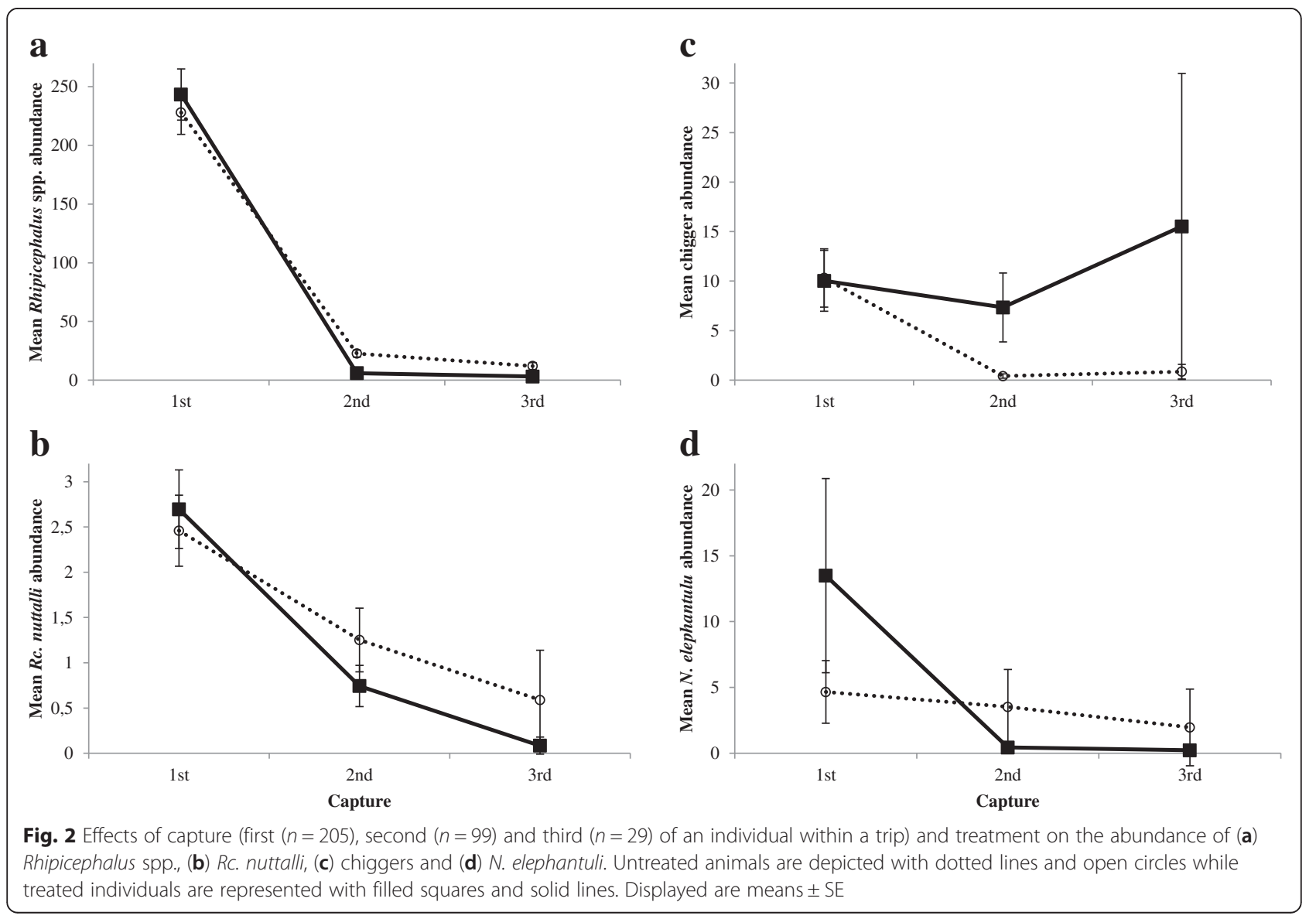




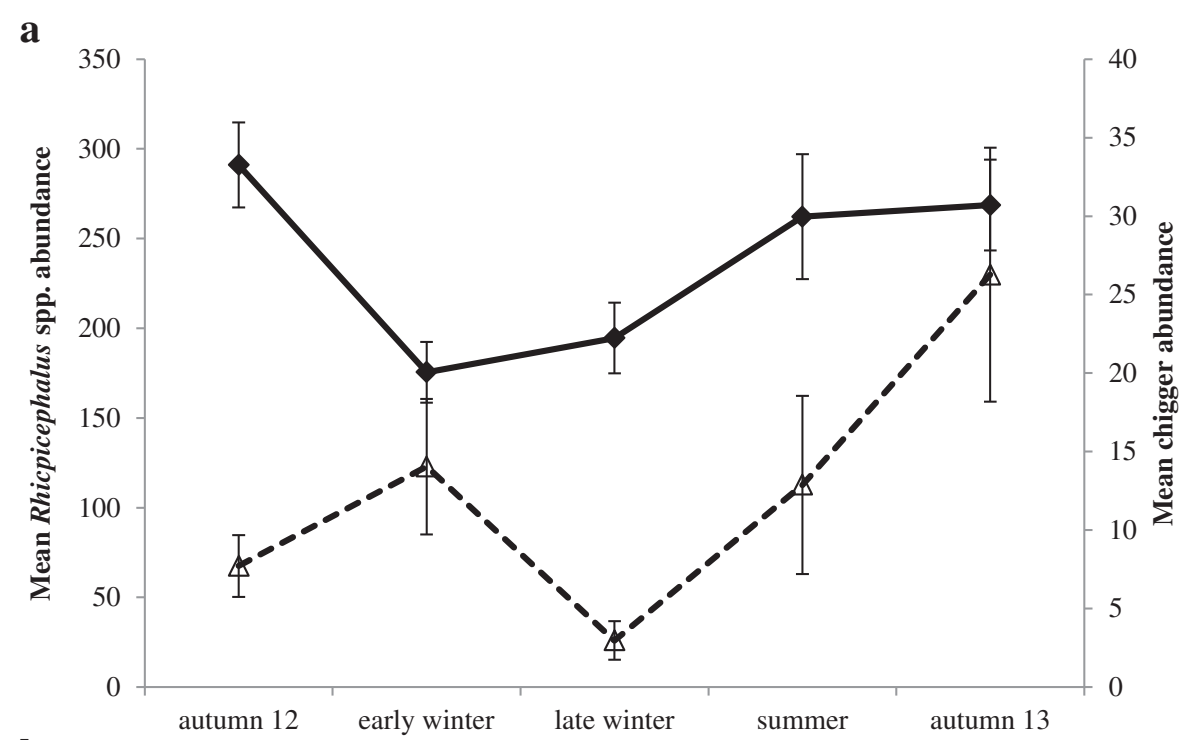

b

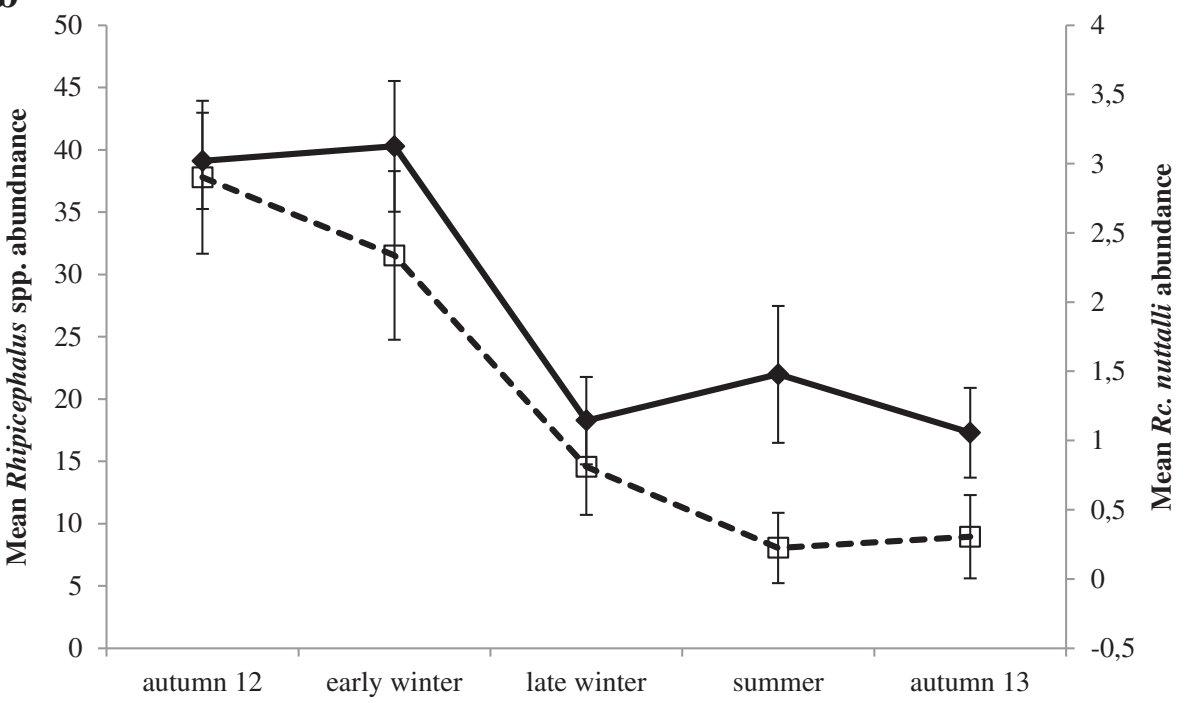

Fig. 3 Seasonal variation in (a) the long-term abundance of Rhipicephalus spp. (black line, filled diamonds) and chiggers (dashed line, open triangles) and (b) the short-term abundance of Rhipicephalus spp. and Rc. nuttalli (dashed line, open squares) sustained by E. myurus during the study period. Displayed are means \pm SE

during autumn 12 and early winter compared to the other seasons (LSD: $p \leq 0.026$, Fig. $3 b$ ). None of the remaining pairwise comparisons was significant (LSD: $\mathrm{p} \geq 0.170$ ).

For short-term data the interaction between season and capture was significant for the abundance of Rhipicephalus spp. (Table 2). It was significantly higher for first compared to both second and third captures during all seasons (LSD: $p<0.0001$ for all, Additional file 1: Figure S6). In contrast, it was significantly lower for third compared to second captures in autumn 12 and late winter (LSD: $\mathrm{p} \leq 0.029$, Additional file 1: Figure S6) but not the other seasons (LSD: $\mathrm{p} \geq 0.117$ ). In addition, it was significantly lower in early winter compared to autumn 12 and 13 (LSD: $\mathrm{p} \leq 0.019)$ and late winter compared to autumn 12 (LSD: $p=0.017$, Additional file 1: Figure S6) for first captures. Conversely, it was significantly higher in autumn 12 and early winter compared to all other seasons for second captures (LSD: $\mathrm{p} \leq 0.010$, Additional file 1: Figure S6) while no other pairwise comparisons were significant. Among third captures the abundance of Rhipicephalus spp. was significantly lower in late winter compared to autumn 12 and early winter (LSD: $\mathrm{p} \leq 0.014$, Additional file 1: Figure S6). In addition, it was significantly lower in summer compared to autumn 12 (LSD: $p=0.011$ ) and early winter compared to autumn 12 (LSD: $p=0.015$, Additional file 1: Figure S6), while no other pairwise comparison was significant (LSD: $\mathrm{p} \geq 0.063$ ). 


\section{Discussion}

Using a combination of observational data and experimental manipulation we provide one of the first studies demonstrating interspecific interactions within the ectoparasite community of a small mammal. Similar to what has previously been reported for endoparasite communities of small mammals [17, 19] we found evidence for competitive interactions between parasite taxa. Furthermore, our data indicate high recruitment rates for the two most prevalent and abundant ectoparasite species suggesting high host exposure. This contrasts strongly with the findings of another recent study on sengis that reported low recruitment rates for the same two ectoparasite species [43]. A number of reasons may account for these differences. Firstly, the sampling regimen differed slightly between both studies with only about half the number of traps used in [43] and traps being opened over four consecutive nights. Also, the study site of the current study and the locality studied in [43], which is located ca. $300 \mathrm{~km}$ south in South Africa's Gauteng province, differ vastly with regards to climate, vegetation cover and small mammal community composition with Goro Game Reserve having a much hotter and drier climate with a continuous rocky landscape and scarce vegetation cover and a less diverse small mammal community comprised of four species (three rodents: Micaelamys namaquensis, Aethomys chrysopilus and Acomys spinossissimus; and the study species) [40, 45]. In contrast, at the Gauteng study site rocky outcrops are interspersed in grassland or savannah habitat and the small mammal community is much more diverse (nine rodents and two insectivores including the study species [32]. For host specialists such as $R$. warburtoni, the lower abundance of sengis may account for the observed differences between the studies. However, this cannot account for the differences in chigger recruitment and although the prevalence of chiggers was comparable in both sites it was almost one order of magnitude lower in the current study compared to [43]. Unlike $R$. warburtoni chiggers are not arid specialists and the lack of vegetation cover may result in a more severe risk of desiccation in our study site. At the same time, the greater host diversity in the Gauteng site may sustain larger numbers of this generalist parasite.

\section{Evidence for competitive interactions and possible mechanisms mediating competition}

We found that treated sengis exhibited an almost fourfold reduction in Rhipicephalus spp. recruitment that coincided with an18-fold increase in chigger recruitment immediately following treatment with an acaricide. At the same time, despite the lack of long-term treatment effects on Rhipicephalus spp. the abundance of lice increased more than six-fold over the study period in treated but not untreated individuals suggesting a between-taxa competition. For long-term data the species richness was lowest when the abundance of Rhipicephalus spp. was greatest corroborating the hypothesis that dominant or keystone species strongly influence ecological communities [24].

The different temporal patterns observed for chiggers and lice, respectively, suggest that the mechanisms mediating the competitive relationship with Rhipicephalus spp. differs for the two taxa. Since both ticks and N. elephantuli are haematophagous, indirect competition for resources could account for the increase in louse burden [6]. However, competition for resources is unlikely to explain the relationship between these ticks and chiggers since chiggers feed on liquefied epithelial cells and tissue [44]. Thus, the high abundance and prevalence of Rhipicephalus spp. and chiggers could suggest a direct competition for attachment sites. The vast majority of Rhipicephalus spp. were $R$. warburtoni and sengis are their preferred host [33]. On sengis this tick is predominately found on the ridges of the ears and the base of the lower back while chiggers are attached to the lower back only. In contrast, in sympatric rodents, where $R$. warburtoni is absent, chiggers are usually found on the ears [43] supporting this hypothesis. Similar competition for attachment sites between ticks and mites has been suggested for Ixodes pacificus and chiggers parasitizing lizards [46] further corroborating our hypothesis. Since competition for attachment sites has been reported between co-infecting ticks species $[25,26]$ and our treatment would have targeted all tick species, we cannot entirely exclude the possibility that such a competitive relationship may also exist between Rhipicephalus spp. and Rc. nuttalli. At the same time, the more than 30 times greater abundance of Rhipicephalus spp. compared to Rc. nuttalli makes it unlikely that the removal of $R c$. nuttalli alone would have resulted in similar increases of chiggers and lice.

Evidence for a strong competitive interaction between the dominant tick species and chiggers exploiting sengis has previously been reported for the study species and thus appears to be consistent regardless of differences in climate, host density or small mammal community composition. In contrast, evidence for interspecific interactions between co-infecting ectoparasite species was reported in the Gauteng sengi population with prevalences or abundances of several less prevalent and/or abundant tick species increasing as a result of the treatment while others appear to respond to other ectoparasite taxa. At the same time, although lice were found in the Gauteng population no long-term treatment effects such as reported in the current study were found [43]. This may be partially linked to the more diverse ectoparasite community recorded for the more southern 
sengi population that comprises at least eleven tick species of which four exceed prevalences of $15 \%$, chiggers, $N$. elephantuli as well as five flea species [43]. This may also account for the differences in the distribution of certain ectoparasite taxa and although the prevalence and abundance of the dominant tick species were comparable between both populations this did not apply to the remaining most common ectoparasite taxa found in the current study. In addition, the previous study was conducted over a longer time period (3 years) than the current study and may thus have been able to pick up additional, more subtle relationships such as those between rarer tick species. It furthermore suggests that caution should be applied when attempting to generalize the findings from one host population to others.

\section{The role of parasite life-history traits in interspecific competition within the ectoparasite community}

The contrasting temporal patterns in the competitive interactions between Rhipicephalus spp. and lice and chiggers, respectively, could be linked to the differences in host exposure to $N$. elephantuli and chiggers as well as differences in life-history traits between these two species. While only the larval stage of chiggers are parasitic and our recruitment data suggest that they are present in the environment throughout the year, lice spend their entire life-cycle on the host $[30,44]$. Consequently, chigger recruitment would largely be determined environmentally, while the recruitment of lice depended mostly on lice hatched from the eggs that remained on the sengis after each capture [30, 47]. Given the extremely high abundance of $R$. warburtoni among first captures, a substantially reduced feeding competition could be expected for several weeks as a result of our treatment. This is likely to have led to greater reproductive success once these lice reached the adult stage and could account for the overall increase in $N$. elephantuli abundance. However, unlike with the permanently questing chigger larvae present in the environment, the developmental time meant that this effect would only be apparent with a certain time delay.

Variables affecting community responses to perturbations It has been suggested that the nature of interspecific interactions determines the resilience of a community [21-23]. Our finding that the duration of treatment effects differed between ectoparasite taxa which compete via different mechanisms (i.e. direct vs. indirect) provides corroborating evidence for this hypothesis. In addition, chiggers and lice differ markedly in their lifehistory traits and this suggests that variation in lifehistory strategies of non-target members of the parasite community should be taken into account when targeted treatment is considered for management purposes. The importance of such context-dependent approaches to avoid undesirable infracommunity responses has previously been highlighted for the epidemiology of endoparasite communities $[9,10,17]$.

Although our results suggest competitive interactions between the most common and abundant ectoparasite, Rhipicephalus spp., for both chiggers and lice the divergent temporal responses of these two species to the removal of Rhipicephalus spp. do not lend support to the hypothesis that community resilience will be lower if perturbations affect keystone or dominant species with many interactions in the community [24]. Instead the mechanisms governing resilience appear to be more complex for ectoparasite communities and both lifehistory as well as interaction mechanism may contribute to the community response to the removal of dominant species.

\section{Contributions of other factors to the distribution of ectoparasites}

Our analyses revealed seasonal fluctuations for ticks and chiggers that quest from the environment. This is in accordance with the notion that ectoparasites that spend a large proportion of their life-cycle off-host are generally assumed to be predominately affected by environmental rather than host factors [30, 40, 48, 49]. In contrast, the lack of seasonal effects observed for $N$. elephantuli are probably a result of the fact that lice spend their entire life-cycle on the host and are thus more dependent on 'host microclimate' than environmental factors [30, 47]. However, seasonal differences in recruitment rates were only apparent for Rhipicephalus spp. suggesting that apart from environmental and host factors [40, 41] interspecific interactions within the infracommunity may play a role in generating the seasonal patterns observed in this and other studies. This hypothesis deserves further attention in future studies.

\section{Conclusion}

We found evidence for several competitive interspecific interactions between members of the ectoparasite infracommunity of sengis. However, the competitive mechanisms mediating these interactions are likely to differ between taxa. In addition, long-term effects of our experimental perturbation were observed for lice, while they were only of short duration for chiggers indicating that in addition to the nature of community interactions, parasite life-history may affect community resilience. It furthermore suggests that the application of common anti-parasite treatments targeting particular parasite groups can also affect non-target parasites. 


\section{Ethical approval}

This research was conducted under permit number 001CPM401-0002 from the Department of Environmental Affairs, Limpopo Province, Africa and approved by the Ethics committee of the University of Pretoria (EC11-12).

\section{Additional file}

Additional file 1: Supplementary material. Evidence for interspecific interactions in the ectoparasite infracommunity of a wild mammal. (PDF $646 \mathrm{~kb}$ )

\section{Competing interests}

The authors declare that they have no competing interests.

\section{Authors' contributions}

Conceived and designed study: HL. Data collection and parasite identification: $\mathrm{SH}, \mathrm{IGH}$, Performed statistical analyses: $\mathrm{SH}$ and $\mathrm{HL}$, Contributed materials/analysis tools: NCB, IGH, Wrote the manuscript: $\mathrm{SH}, \mathrm{HL}, \mathrm{IGH}$ and NCB. All authors read and approved the final manuscript.

\section{Acknowledgements}

We are greatly indebted to the management and staff of the Goro Game Reserve, especially D. Dewsnap. We are furthermore grateful for the help of numerous volunteers for their assistance in the lab and in the field. This work was supported by NRF grants to SH and IGH as well as the DST-NRF SARChl Chair of Behavioural Ecology and Physiology to NCB and funding by the University of Pretoria to HL.

\section{Author details}

${ }^{1}$ Department of Zoology and Entomology, University of Pretoria, Private Bag X20, Hatfield 0028, South Africa. ${ }^{2}$ Department of Veterinary Tropical Diseases, Faculty of Veterinary Science, University of Pretoria, Private Bag X04,

Onderstepoort 0110, South Africa.

\section{Received: 6 October 2015 Accepted: 26 January 2016}

BP

\section{References}

1. Poulin R. Evolutionary Ecology of Parasites. Princeton: Princeton University Press; 2007.

2. Wilson K, Bjørnstad ON, Dobson AP, Merler S, Poglayen G, Randolph SE, et al. Heterogeneities in macroparasite infections: patterns and processes. In: Hudson PJ, Rizzoli A, Grenfell BT, Heesterbeek H, Dobson AP, editors. Ecol Wildl Dis. New York: Oxford University Press; 2002. p. 6-44.

3. Petney TN, Andrews RH. Multiparasite communities in animals and humans: frequency, structure and pathogenic significance. Int J Parasitol. 1998;28: 377-93.

4. Cox FE. Concomitant infections, parasites and immune responses. Parasitology. 2001;122:S23-38.

5. Graham AL. Ecological rules governing helminth-microparasite coinfection. Proc Natl Acad Sci U S A. 2008;105:566-70.

6. Pedersen $A B$, Fenton $A$. Emphasizing the ecology in parasite community ecology. Trends Ecol Evol. 2007;22:133-9.

7. Telfer S, Lambin X, Birtles R, Beldomenico P, Burthe S, Paterson S, et al. Species interactions in a parasite community drive infection risk in a wildlife population. Science. 2010;330:243-6.

8. Randall J, Cable J, Guschina IA, Harwood JL, Lello J. Endemic infection reduces transmission potential of an epidemic parasite during co-infection. Proc R Soc B. 2013;280:20131500.

9. Griffiths EC, Pedersen AB, Fenton A, Petchey OL. Analysis of a summary network of co-infection in humans reveals that parasites interact most via shared resources. Proc R Soc B. 2014;281:20132286.

10. Fenton A. Dances with worms: the ecological and evolutionary impacts of deworming on coinfecting pathogens. Parasitology. 2013;140:1119-32.

11. Knowles SCL. The effect of helminth co-infection on malaria in mice: a meta-analysis. Int J Parasitol. 2011;41:1041-51.

12. Bush AO, Lafferty KD, Lotz JM, Shostak AW. Parasitology meets ecology on its own terms: Margolis et al. revisited. J Parasitol. 1997;83:575-83.
13. Lello J, Boag B, Fenton A, Stevenson IR. Competition and mutualism among the gut helminths of a mammalian host. Nature. 2004:428:20-4.

14. Ezenwa VO, Etienne RS, Luikart G, Beja-Pereira A, Jolles AE. Hidden consequences of living in a wormy world: nematode-induced immune suppression facilitates tuberculosis invasion in African buffalo. Am Nat. 2010;176:613-24.

15. Moreno PG, Eberhardt MAT, Lamattina D, Previtali MA, Beldomenico PM Intra-phylum and inter-phyla associations among gastrointestinal parasites in two wild mammal species. Parasitol Res. 2013;112:3295-304.

16. Fenton A, Viney ME, Lello J. Detecting interspecific macroparasite interactions from ecological data: patterns and process. Ecol Lett. 2010;13:606-15

17. Pedersen $A B$, Antonovics J. Anthelmintic treatment alters the parasite community in a wild mouse host. Biol Lett. 2013;9:20130205.

18. Johnson PTJ, Buller ID. Parasite competition hidden by correlated coinfection: using surveys and experiments to understand parasite interactions. Ecology. 2011:92:535-41.

19. Knowles $\mathrm{SCL}$, Fenton A, Petchey $\mathrm{OL}$, Jones TR, Barber R, Amy B, et al. Stability of within-host - parasite communities in a wild mammal system. Proc R Soc B. 2013;280:20130598.

20. Fenton A, Knowles SCL, Petchey $\mathrm{OL}$, Pedersen $\mathrm{AB}$. The reliability of observational approaches for detecting interspecific parasite interactions: comparison with experimental results. Int J Parasitol. 2014;44:437-45.

21. Pimm SL. The complexity and stability of ecosystems. Nature. 1984;307:321-6.

22. Neutel A-M, Heesterbeek J, de Ruiter P. Stability in real food webs: weak links in long loops. Science. 2002;296:1120-3.

23. Stouffer DB, Bascompte J. Compartmentalization increases food-web persistence. Proc Natl Acad Sci. 2011;108:3648-52.

24. Solé RV, Montoya JM. Complexity and fragility in ecological networks. Proc R Soc B. 2001;268:2039-45

25. Andrews R, Petney T. Competition for sites of attachment to hosts in three parapatric species of reptile tick. Oecologia. 1981;51:227-32

26. Andrews RH, Petney TN, Bull CM. Niche changes between parasite populations: an example from ticks on reptiles. Oecologia. 1982;55:77-80.

27. Krasnov BR, Stanko M, Morand S. Are ectoparasite communities structured? Species co-occurrence, temporal variation and null models. J Anim Ecol. 2006;75:1330-9.

28. Sonenshine D. Biology of Ticks. Oxford: Oxford University Press; 1991.

29. Owen JP, Nelson AC, Clayton DH. Ecological immunology of bird-ectoparasite systems. Trends Parasitol. 2010;26:530-9.

30. Marshall AG. The Ecology of Ectoparasitic Insects. London: Academic; 1981.

31. Skinner JD, Chimimba CT. The Mammals of the Southern African Subregion. Cambridge: Cambridge University Press; 2005.

32. Fagir DM, Ueckermann EA, Horak IG, Bennett NC, Lutermann H. The Namaqua rock mouse (Micaelamys namaquensis) as a potential reservoir and host of arthropod vectors of diseases of medical and veterinary importance in South Africa. Parasit Vectors. 2014;7:366.

33. Fagir DM, Horak IG, Ueckermann EA, Bennett NC, Lutermann H. Ectoparasite diversity in the eastern rock sengis (Elephantulus myurus): The effect of seasonality and host sex. African Zool. 2015:50:109-17.

34. Cochet P, Birckel P, Bromet-Petit M, Bromet N, Weil A. Skin distribution of fipronil by microautoradiography following topical administration to the beagle dog. Eur J Drug Metab Pharmacokinet. 1997;22:211-6.

35. Metzger ME, Rust MK. Laboratory evaluation of fi pronil and imidacloprid topical insecticides for the control of the plague vector Oropsylla montana (Siphonaptera: Ceratophyllidae) on California ground squirrels (Rodentia: Sciuridae). J Med Entomol. 2002;39:152-61.

36. Smith A, Telfer S, Burthe S, Bennett M, Begon M. A role for vectorindependent transmission in rodent trypanosome infection? Int J Parasitol. 2006;36:1359-66.

37. Hillegass MA, Waterman JM, Roth JD. Parasite removal increases reproductive success in a social African ground squirrel. Behav Ecol. 2010:21:696-700.

38. Scantlebury M, Waterman JM, Hillegass M, Speakman JR, Bennett NC. Energetic costs of parasitism in the Cape ground squirrel Xerus inauris. Proc R Soc B. 2007;274:2169-77.

39. Dolan MC, Maupin GO, Schneider BS, Denatale C, Hamon N, Cole C, et al. Control of immature Ixodes scapularis (Acari: Ixodidae) on rodent reservoirs of Borrelia burgdorferi in a residential community of southeastern Connecticut. J Med Entomol. 2004:41:1043-54. 
40. Lutermann H, Medger K, Horak IG. Abiotic and biotic determinants of tick burdens in the eastern rock sengi (Elephantulus myurus). Med Vet Entomol. 2012;26:255-62.

41. Lutermann H, Medger K, Horak IG. Effects of life-history traits on parasitism in a monogamous mammal, the eastern rock sengi (Elephantulus myurus). Naturwissenschaften. 2012;99:103-10.

42. Symonds M, Moussalli A. A brief guide to model selection, multimode inference and model averaging in behavioural ecology using Akaike's information criterion. Behav Ecol Sociobiol. 2011;65:13-21.

43. Lutermann H, Fagir D, Bennett N. Complex interactions within the ectoparasite community of the eastern rock sengi (Elephantulus myurus). Int J Parasitol Parasites Wildl. 2015;4:148-58.

44. Shatrov AB, Kudryashova NI. Taxonomy, life cycles and the origin of parasitism in trombiculid mites. In: Morand S, Krasnov BR, Poulin R, editors. Micromammals and macroparasites - From evolutionaryecology to management. Tokyo: Springer Verlag; 2006. p. 119-40.

45. Harrison A, Bown KJ, Horak IG. Detection of Anaplasma bovis in an undescribed tick species collected from the eastern rock sengi Elephantulus myurus. J Parasitol. 2011;97:1012-6.

46. Pollock NB, Vredevoe LK, Taylor EN. The effect of exogenous testosterone on ectoparasite loads in free-ranging western fence lizards. J Exp Zool. 2012:317:447-54

47. Stanko M, Fricová J, Miklisová D, Khokhlova IS, Krasnov BR. Environment-related and host-related factors affecting the occurrence of lice on rodents in Central Europe. Parasitology. 2015;142:938-47.

48. Needham GR, Teel PD. Off-host physiological ecology of ixodid ticks. Annu Rev Entomol. 1991;36:659-81.

49. Benoit JB, Denlinger DL. Meeting the challenges of on-host and off-host water balance in blood-feeding arthropods. J Insect Physiol. 2010;56:1366-76.

\section{Submit your next manuscript to BioMed Central and we will help you at every step:}

- We accept pre-submission inquiries

- Our selector tool helps you to find the most relevant journal

- We provide round the clock customer support

- Convenient online submission

- Thorough peer review

- Inclusion in PubMed and all major indexing services

- Maximum visibility for your research

Submit your manuscript at www biomedcentral.com/submit

C Biomed Central 